\title{
Biosynthesis of Providencin: Understanding Photochemical Cyclobu- tane Formation with Density Functional Theory
}

\author{
Bencan Tang ${ }^{a^{*}}$ and Robert S. Paton ${ }^{b, c^{*}}$ \\ ${ }^{a}$ Department of Chemical and Environmental Engineering, Faculty of Science and Engineering, The University of Nottingham Ningbo China, 199 Taikang \\ East Road, Ningbo 315100, China; ${ }^{b}$ Chemistry Research Laboratory, University of Oxford, 12 Mansfield Road, Oxford OX1 3TA, UK. ${ }^{c}$ Department of \\ Chemistry, Colorado State University, Fort Collins, CO 80523, USA. \\ *Bencan.Tang@nottingham.edu.cn, Robert.Paton@colostate.edu
}

Supporting Information Placeholder
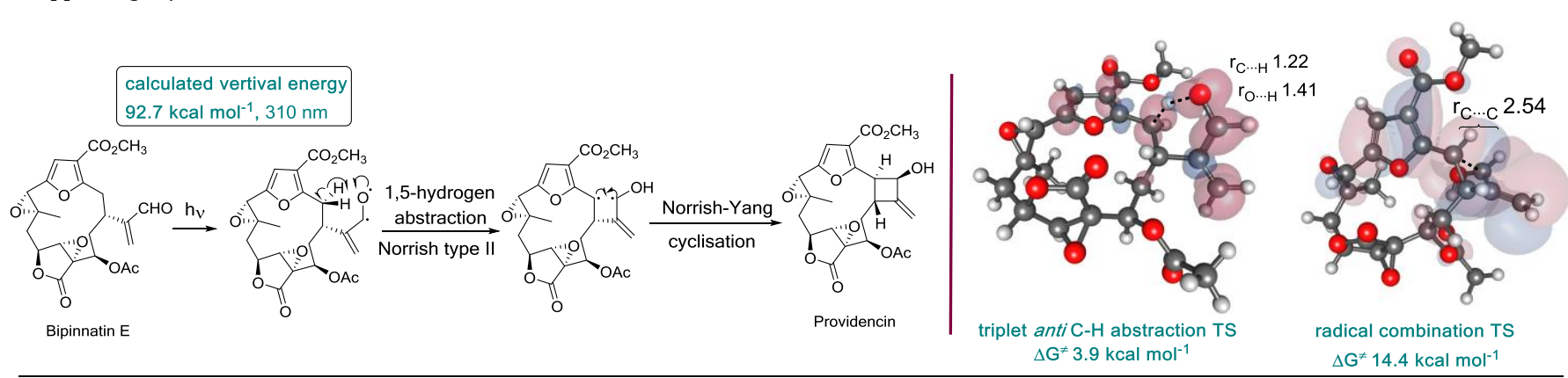

ABSTRACT: The unique structure of furanocembranoid natural product providencin has stimulated biosynthetic hypotheses, especially concerning the formation of its cyclobutanol ring. One such hypothesis involves a photochemically-induced Norrish-Yang cyclisation in bipinnatin E. We have used computation to assess the feasibility and the stereochemical outcome of this proposed biosynthetic transformation. Density functional theory calculations reveal that the proposed Norrish-Yang cyclisation in bipinnatin E is possible, and that the stereoselectivity of this step is consistent with that of the natural product.

Isolated from Caribbean sea plume Pseudopterogorgiakallos, providencin 1 belongs to the family of furanocembranoid natural products, which contain a 14-membered macrocycle, a 2,3,5-trisubstituted furan, and a butenolide ring as common features, e.g. bipinnatin E (2) and bipinnatin J (3). ${ }^{1}$ Compared to other furanocembranes, the most intriguing part of this diterpenoid is the methylene-cyclobutanol fused at the C1 and C2-positions (Figure 1). Moreover, the trans arrangement of the $\Delta-7,8$ epoxide, together with the rigid angle between $\mathrm{C} 2$ and $\mathrm{C} 7$ appendages around the furan ring, make the macrocycle highly strained. The relative configuration was determined by X-ray crystallography; however the absolute configuration is still unknown. ${ }^{1}$ Providencin exhibits modest activity against human breast, lung and CNS cancer cell lines. The biological profile and structural complexity of this marine diterpenoid have inspired several synthetic approaches towards the construction of the macrocycle ring as well as the furanyl-cyclobutanol moiety. ${ }^{2-6}$ Nevertheless, the total synthesis of providencin has not yet been accomplished. The synthetic challenge lies in its structural features: 1) the highly strained 14-membered macrocyclic structure - it has been observed that it is almost impossible to build a Dreiding model without breaking any bond; 2 2) the highly functionalized cyclobutanol moiety, with three contiguous stereo centers in addition to the exo-methylene substitution.
Biosynthetic proposals for the genesis of Providencin from other simple furanocembranes, i.e. bipinnatin J and E, which were also found in Pseudopterogorgia, ${ }^{7}$ have been reported. ${ }^{2,6}$ Pattenden pro-

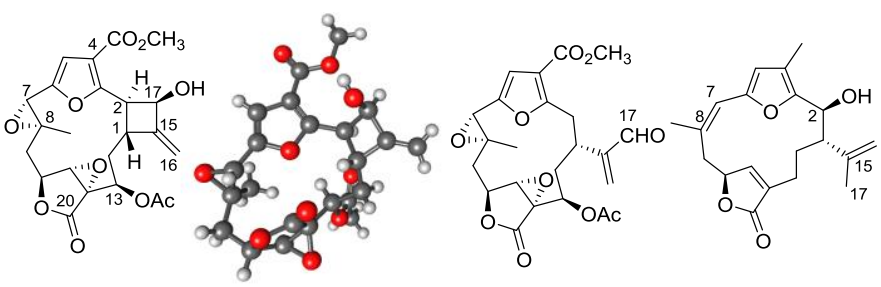

1, Providencin $\quad X$-ray structure of $1 \quad 2$, Bipinnatin $E \quad 3$, Bipinnatin $J$

Figure 1. Providencin 1, bipinnatin $\mathrm{E}(2)$ and $\mathrm{J}(3)$

posed a Norrish-Yang cyclisation ${ }^{8,9}$ forms the furan methylene cyclobutanol moiety in Providencin (Scheme 1a), based on a photochemically mediated intramolecular $\mathrm{C}-\mathrm{H}$ insertion reaction from the $\alpha, \beta$-unsaturated aldehyde in bipinnatin E. ${ }^{6}$ Although the Norrish-Yang cyclisation is rare in natural product biosynthesis $^{10-15}$, photochemical reactions play an important role in natural product biosynthesis. ${ }^{16}$ Many cyclobutanes are thought to arise from photochemical reactions. ${ }^{17}$ Mulzer proposed that the cyclobutane ring in providencin arises from a cationic cyclization in C-2 dehydroxylated bipinnatin J, followed by C7-C8 E/Z isomerization and further oxidation at $\mathrm{C} 17$ to generate the $\mathrm{C} 17$ hydroxyl group in providencin (Scheme 1b). ${ }^{2}$ 


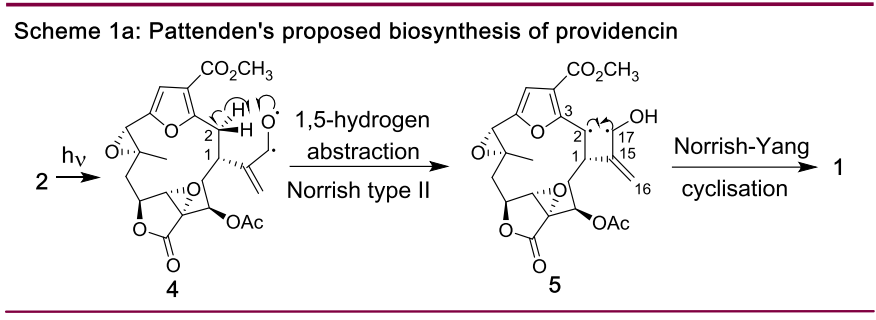

Scheme 1b. Mulzer's proposal for the biosynthetic formation of providencin

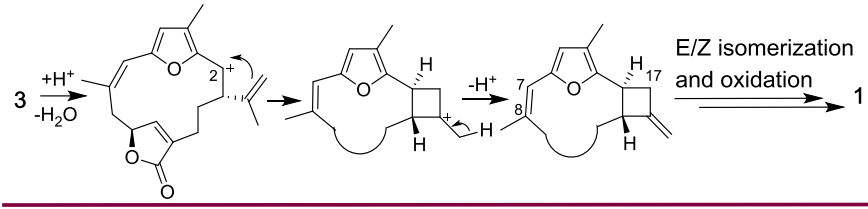

Scheme 2: Pattenden's model study for the proposed biosynthesis of providencin

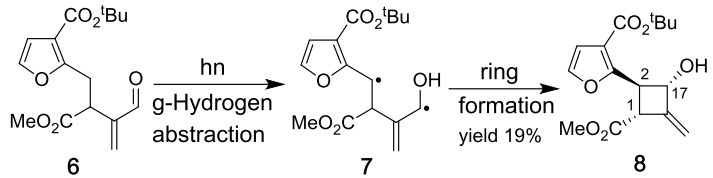

Pattenden and coworkers performed a synthetic model study of the proposed Norrish-Yang cyclisation in bipinnatin E (2). ${ }^{6}$ Irradiation of a solution of $\alpha, \beta$-unsaturated aldehyde 6 in benzene in a pyrex photoreactor using light from a $400 \mathrm{~W}$ medium pressure Hg lamp, with regular monitoring by ${ }^{1} \mathrm{H}$ NMR spectroscopy, led to methylenecyclobutanol 8 through the anticipated NorrishYang reaction. The isolated yield was $19 \%$. The conversion of 6 into 8 demonstrates the plausibility of photochemically-mediated cyclobutanol formation as found in the structure of providencin $1 .{ }^{6}$ However, the stereochemistry of 8 has a trans-1,2-trans-2,17. relationship around the cyclobutane ring, in contrast to the trans1,2-cis-2,17-relationship in providencin. It was anticipated that the conformational bias imposed by the macrocyclic ring of thefuranocembrane precursor, such as bipinnatin $\mathrm{E}$, may influence diastereoselectivity towards the product, following a photochemical C-H insertion reaction similar to the conversion of 6 to $8 .{ }^{6}$ Therefore, further understanding of the nature of this reaction in the origin of providencin is necessary.

Quantum chemical calculations have been widely used to uncover new mechanisms, ${ }^{18,19}$ as well as seeking theoretical support to biosynthetic proposals. ${ }^{20-22}$ For instances, calculations have shown the oxidopyrilium-alkene cycloaddition in the biomimetic synthesis of intricarene $e^{23,24}$ is feasible, with activation barriers around $20 \mathrm{kcal} \cdot \mathrm{mol}^{-1} .{ }^{25}$ Quantum chemical calculations at CASSCF/CASPT2 and DFT/TD-DFT level of theory also enabled the rationalization of complex reaction pathways involving multiple excited states in the photo-induced intricarene formation. ${ }^{26}$ Transannular cycloaddition reactions of furanoxonium ions have been conducted both experimentally ${ }^{27,28}$ and investigated computationally with density functional theory (DFT) in furanocembrane biosynthesis. ${ }^{29}$ A recent DFT study of cyclobutane formation in Bielschowskysin biosynthesis predicted both thermal closure and photocycloaddition are possible, with the latter being more efficient. ${ }^{30}$ Herein we report computational studies to assess the feasibility and stereochemistry outcome of the aforementioned Norrish-Yang cyclisation in the cyclobutanol formation in the biosynthesis of 1 . $\mathrm{n}-\pi^{*}$ Photoexcitation and $\mathrm{H}$-abstraction. The aforementioned Norrish-Yang reaction is initiated by $n-\pi^{*}$ excitation of the carbonyl group in ketones or aldehydes, i.e. the carbonyl group in the $\alpha, \beta$-unsaturated aldehydes in bipinnatin E (2) (Scheme 1a). The key step is the intramolecular 1, 5- $\mathrm{H}$ migration from the $\gamma$ arbon atom (C2 in 4) to the oxygen atom of the carbonyl group (Scheme 1a: $2 \rightarrow 4 \rightarrow 5$ ). As a result, a biradical intermediate is formed. This biradical species can then either cyclize to the corresponding cyclobutanol (Yang cyclization, $5 \rightarrow 1$ ) or undergo fragmentation (Norrish type II cleavage). ${ }^{8,9}$

Upon photo-excitation, one electron of the oxygen lone pair is promoted to the $\pi^{*}$ orbital of the $\mathrm{C}=\mathrm{O}$ bond. The computed absorption bands for the singlet $\mathrm{n}-\pi^{*}$ excitation, calculated by timedependent (TD)-DFT using CAM-b3lyp/6-311+G(d,p) $)^{31}$ with a conductor-like polarizable continuum model (CPCM) of water, is $92.7 \mathrm{kcal} \cdot \mathrm{mol}^{-1}$, this corresponds to wavelength of $310 \mathrm{~nm}(\mathbf{F i g}$ ure 2). The vertical excitation energy is comparable to similar photo-excitations $\left(98.1 \mathrm{kcal} \cdot \mathrm{mol}^{-1}\right)$ reported in literature, ${ }^{32}$ and the calculated irradiation wavelength is in the range of experiment reported wavelength for aldehyde or ketone $\mathrm{n}-\pi^{*}$ excitation. For example, it is reported that aliphatic aldehydes exhibit a weak absorption band in the wavelength range $240-360 \mathrm{~nm}$ as a result of a symmetry forbidden $n-\pi^{*}$ transition. ${ }^{33}$ The R-band of acrolein is with low density and located in the range of $300-340 \mathrm{~nm}$ range. ${ }^{34}$

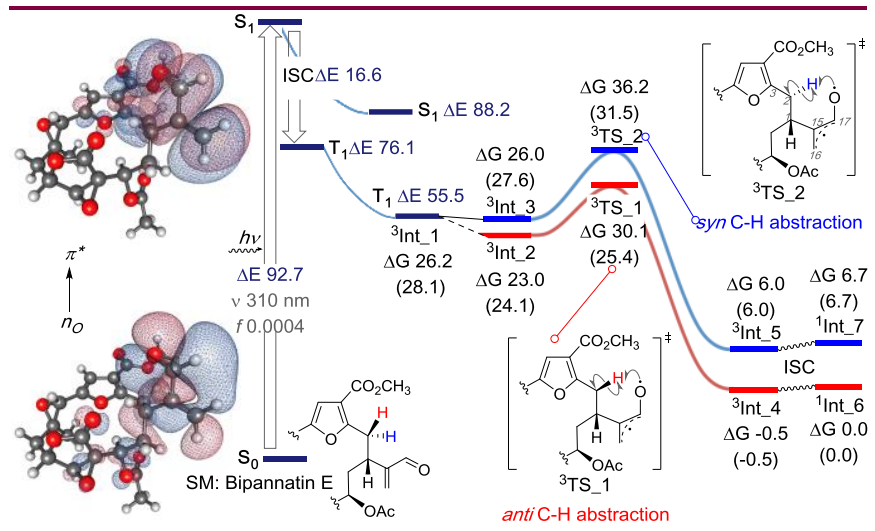

Figure 2. photo initiated $n->\pi^{*}$ excitation and the following hydrogen abstraction in bipinnatin $E$.

All other calculations were performed within the framework of Density Functional Theory (DFT). ${ }^{31}$ Unrestricted Kohn-Sham calculations were used to describe all open-shell species without symmetry constraints. Optimizations were carried out at the (U) B3LYP/6-31G(d) level of theory and single point energies were further estimated using a valence triple- $\zeta$ polarized $6-311+G(d, p)$ basis set, including a conductor-like polarizable continuum model (CPCM) of water. And the (U)MPWB1K and (U)B3LYP functionals were used, in the latter case with an atom-pairwise empirical correction for dispersion (DFT-D3). Intersystem crossing (ISC) from ${ }^{1} n-\pi^{*}$ to ${ }^{3} n-\pi^{*}$ ISC is followed by conformational relaxation to the stable excited state ${ }^{3}{ }^{3} n_{-} 1$. The followed hydrogen abstraction from C2 may occur in an anti or syn fashion as there are two diastereotopic hydrogens on C2. This process is controlled by transition structure ${ }^{3}$ TS-1 (for anti-hydrogen ab- 
straction) and ${ }^{3}$ TS_2 (for syn hydrogen abstraction) with free energies 3.9 and $10.0 \mathrm{kcal} \cdot \mathrm{mol}^{-1}$ above ${ }^{3}$ Int_1 respectively. In the two $\mathrm{H}$-abstraction TSs the distance between oxygen atom and the hydrogen atom is $1.41 \AA$ and $1.38 \AA$ respectively, and the distance between carbon atom and hydrogen atom is $1.22 \AA$ and $1.25 \AA$. The resulting biradical intermediate ${ }^{3}$ Int_4 and ${ }^{3}$ Int_5 lie 26.7 and $20.2 \mathrm{kcal} \cdot \mathrm{mol}^{-1}$ lower in free energy compare to ${ }^{3} \mathrm{Int} \_1$, and both of their singlet states ${ }^{1}$ Int_6 6 and ${ }^{1}$ Int_7 7 are calculated with very similar energies within 0.5 and $0.7 \mathrm{kcal} \cdot \mathrm{mol}^{-1}$, respectively, indicating very little interaction between the two unpaired electrons. The biradicals ${ }^{3}$ Int_4 4 and ${ }^{3}$ Int_5 , together with ${ }^{1}$ Int_6 and ${ }^{1}$ Int_ 7 are planar about each radical center.

${ }^{1}$ Int_6 and ${ }^{1}$ Int_7 (or ${ }^{1}$ Int_9, they differ in the different orientation of C17 hydroxyl group, Figures 3 and 4) are different in that, in ${ }^{1}$ Int_6, the C1-C15 bond is rotated to a position where the hydroxyl group can form a hydrogen bond with the C20 carbonyl oxygen of the epoxybutenolide $\left(r_{\mathrm{O} . . . \mathrm{H}}=1.99\right)$, whereas, in ${ }^{1}$ Int_7, C1-C15 bond is rotated to a position where the same hy-

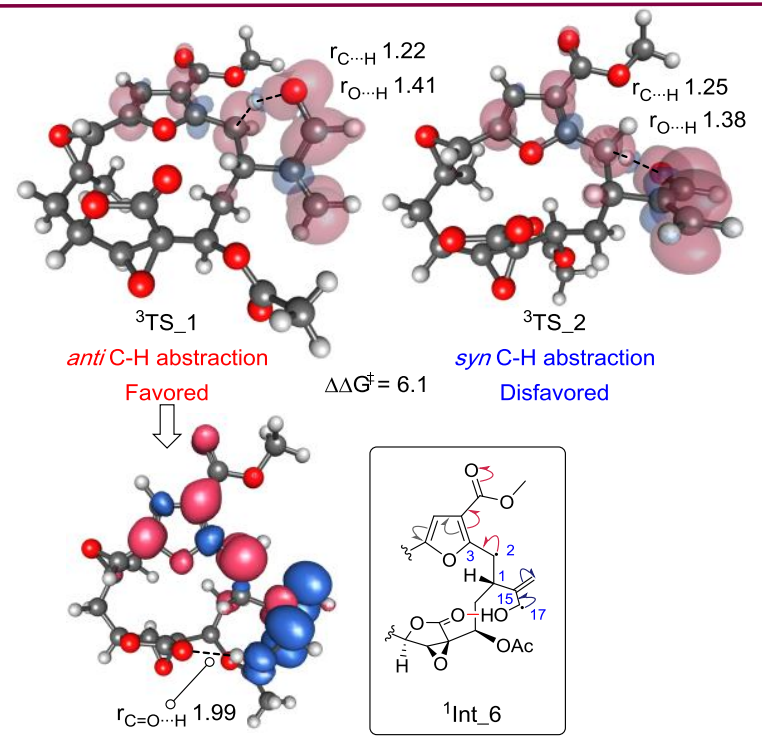

Figure 3. Transition states for $\mathrm{H}$-abstraction in singlet excited bipinnatin $\mathrm{E}$. droxyl group is pointing away from the epoxybutenolide. Moreover, the presence of the very rigid 14-membered ring system with a trans $\Delta-7,8$ epoxide may also play a role in the energy difference between ${ }^{1}$ Int_6 and ${ }^{1}$ Int_7 . As a result, ${ }^{1}$ Int_7 has a higher energy by $6.7 \mathrm{kcal} \cdot \mathrm{mol}^{-1}$. Due to the steric hindrance in the region and the intramolecular bonding in Int_6, we expect C1-C15 rotation for the interconversion between Int_6 and Int_7 is unlikely to happen without any significant barrier at room temperature. Therefore, due to the energy difference in ${ }^{3} \mathrm{TS}_{-} 1$ and ${ }^{3}$ TS_2, i.e. $\Delta \Delta \mathrm{G}^{*} 6.1 \mathrm{kcal} \cdot \mathrm{mol}^{-1},{ }^{1}$ Int_6 is a highly populated intermediate. Nevertheless, we set off to study the radical combination step leading to the formation of the four different diastereoisomers with the understanding that rotation in C1-C15 bond will lead to precursors for different stereochemistry in $\mathrm{C} 2$, hence, Int_6 will form providencin 1 after radical combination through cyclisation ${ }^{1}$ TS_4. ${ }^{1}$ Int_9 is a conformer of ${ }^{1}$ Int_6 due to C1-C15 bond rotation, and leads to a C2, C17 diastereoisomer of providencin (Scheme 3). Rotation of C15-C17 bond in ${ }^{1}$ Int_6 and
${ }^{1}$ Int_9 leads to ${ }^{1}$ Int_8 and ${ }^{1}$ Int_10, which form C17 and C2 epimers of providencin, respectively, following radical combination.

Radical recombination. We set off to understand the four different radical combination reactions starting from singlet biradicals ${ }^{1}$ Int_6 and ${ }^{1}$ Int_7. As shown in Figure 4, ${ }^{1}$ Int_6 is perfectly set for the radial combination to form the desired stereochemistry in Providencin 1 trough ${ }^{1}$ TS_4, the activation Gibbs free energy was calculated as $14.4 \mathrm{kcal} \cdot \mathrm{mol}^{-1}$. Alternatively, C15-C17

Scheme 3. Product diastereoisomers generated from bi-radical conformers due to $\mathrm{C} 1-\mathrm{C} 15$ and C15-C17 bonds rotation in ${ }^{1}$ Int_ 6

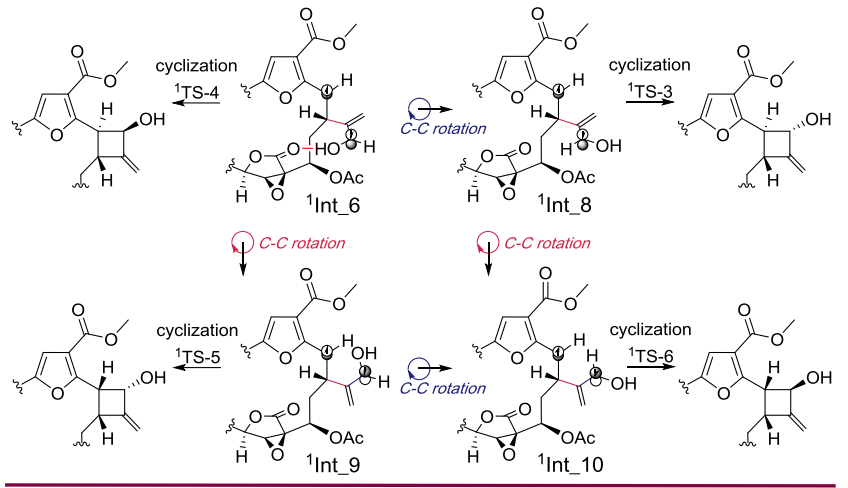

bond rotation in ${ }^{1}$ Int_6 leads to ${ }^{1}$ Int_8 (Figure 4), which was found to be the precursor of the C17-epimer of providencin, after overcoming activation free energy of $7.3 \mathrm{kcal} \cdot \mathrm{mol}^{-1}$ (relative to ${ }^{1}$ Int_6) in the radical combination step through ${ }^{1}$ TS_3. ${ }^{1}$ Int_9 (with a free energy of $7.4 \mathrm{kcal} \mathrm{mol}^{-1}$ ) is found to link with a product with an activation free energy barrier of $12.9 \mathrm{kcal} \mathrm{mol}^{-1}$ of which $\mathrm{C} 2$ and $\mathrm{C} 17$ centres are opposite to the ones in 1. Meanwhile, ${ }^{1}$ Int_10 leads to a C2 epimer of 1 after radical combination. The activation energy of this process is $6.8 \mathrm{kcal} \cdot \mathrm{mol}^{-1}$.

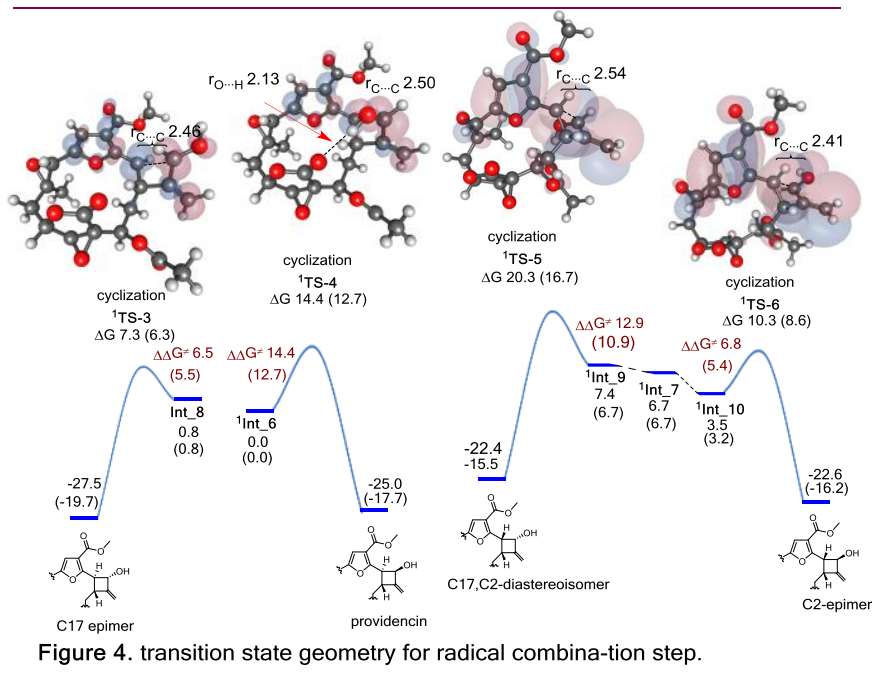

Therefore, without considering the barriers in bond rotations in C1-C15 and C15-C17 bonds, radical combination leading to C17-epimer has the lowest barrier. And this, as mentioned above, is indeed the stereochemistry outcome observed in the model study carried by Pattenden group. ${ }^{6}$ Therefore, based on these data, it is expected that the radical combination leading to the $\mathrm{C} 17$-epimer of providencin has the lowest activation barrier, 
i.e. $7.3 \mathrm{kcal} \mathrm{mol}^{-1}$ (relative to ${ }^{1}$ Int_6), hence the C-17 epimer would be the favored product.

At this stage, several questions arise: (a) does the $\mathrm{C}=\mathrm{C}$ stereochemistry at C7-C8 affect the stereochemical outcome of radical recombination? (b) Could it be possible that in nature, the Norrish-Yang cyclisation reaction happens at an earlier stage, prior to formation of the epoxides on C7-C8 and C11-C12? (c) Does the macrocyclic constraint or the substitution pattern on the furan ring affect this reaction? To answer these questions, we investigated the cyclobutene-forming radical ring-closure of four different substrates (Figure 5). These substrates differ in the substitution of the furan ring and the geometry of the C7-C8 double bond. As shown in the supporting information, the TS leading to the C17 epimer (relative to providencin) is most stable for all five different substrates.

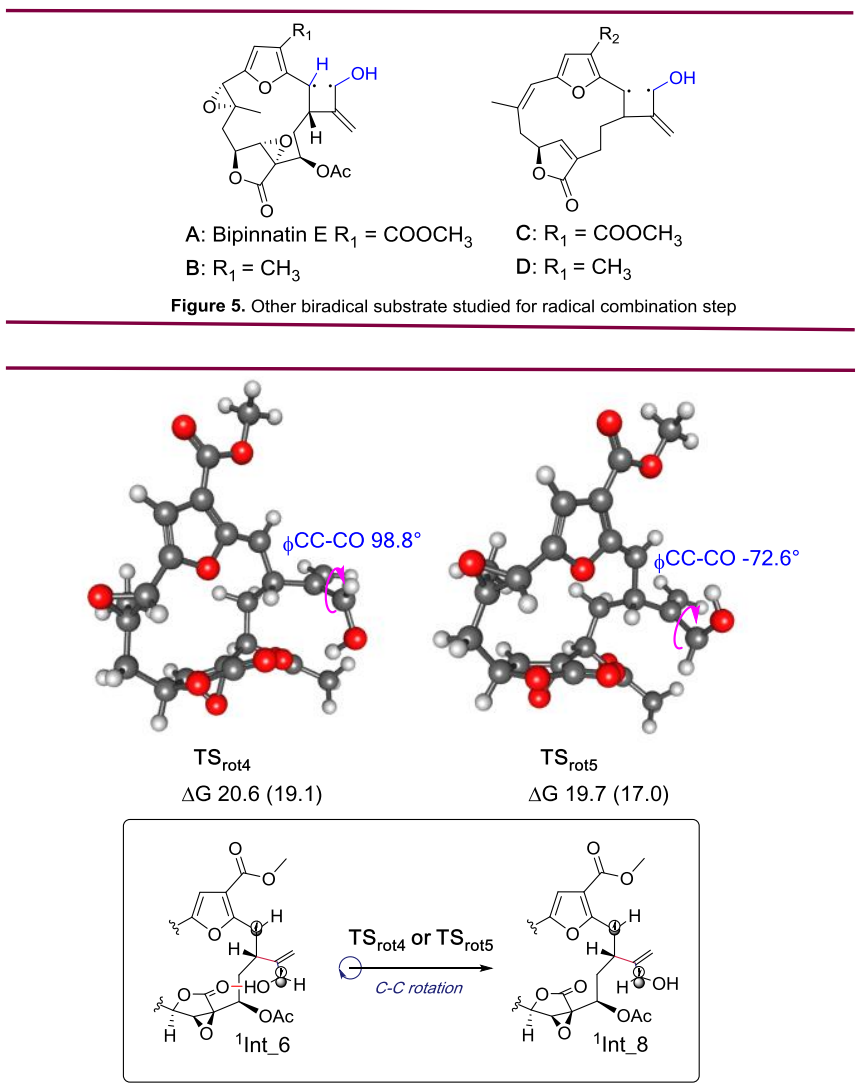

Figure 6. Interconversion of ${ }^{1}$ Int_6 and ${ }^{1}$ Int_8

Interconversion of biradical conformers. Our discussions up to this point have assumed a Curtin-Hammett scenario in which biradical conformers interconvert more quickly than ring-closure. However, due to steric hindrance around C1-C15 and C15$\mathrm{C} 17$ bonds preventing facile interconversion, we began to question this assumption. For instance, in order to access ${ }^{1}$ Int_8 from ${ }^{1}$ Int_6 the C15-C17 bond needs to rotate (Scheme 3), an intermolecular H-bond must be broken and radical allylic conjugation disrupted. Calculated barriers for rotation in a clockwise or anticlockwise fashion are 20.6 and $19.7 \mathrm{kcal} \mathrm{mol}^{-1}$, respectively. This is higher by $5-6 \mathrm{kcal} \mathrm{mol}^{-1}$ than ring-closure (Figure 6). Rotation about the C1-C15 bond was also studied (Figure 7). Again, steric congestion and the energetic cost associated with having to break a stabilizing H-bond result in appreciable barriers for the interconversion of Int_6 to ${ }^{1}$ Int_9: this can occur in a single step (via $\mathrm{TS}_{\text {rot1 }}$ with barrier $17.0 \mathrm{kcal} \cdot \mathrm{mol}^{-1}$ ) or sequentially (through $\mathrm{TS}_{\text {rot2 }}$ and $\mathrm{TS}_{\text {rot3 } 3}$ with barriers of 10.9 and $16.2 \mathrm{kcal} \cdot \mathrm{mol}^{-1}$ ). Our calculations therefore suggest that a non Curtin-Hammett scenario can arise, in which ${ }^{1}$ Int_6 is the only intermediate to undergo cyclization since conformational interconversion is severely restricted. The $\mathrm{C}-\mathrm{H}$ abstraction step becomes stereodetermining, and is set by the geometry of ${ }^{3} \mathrm{TS} \_1$.

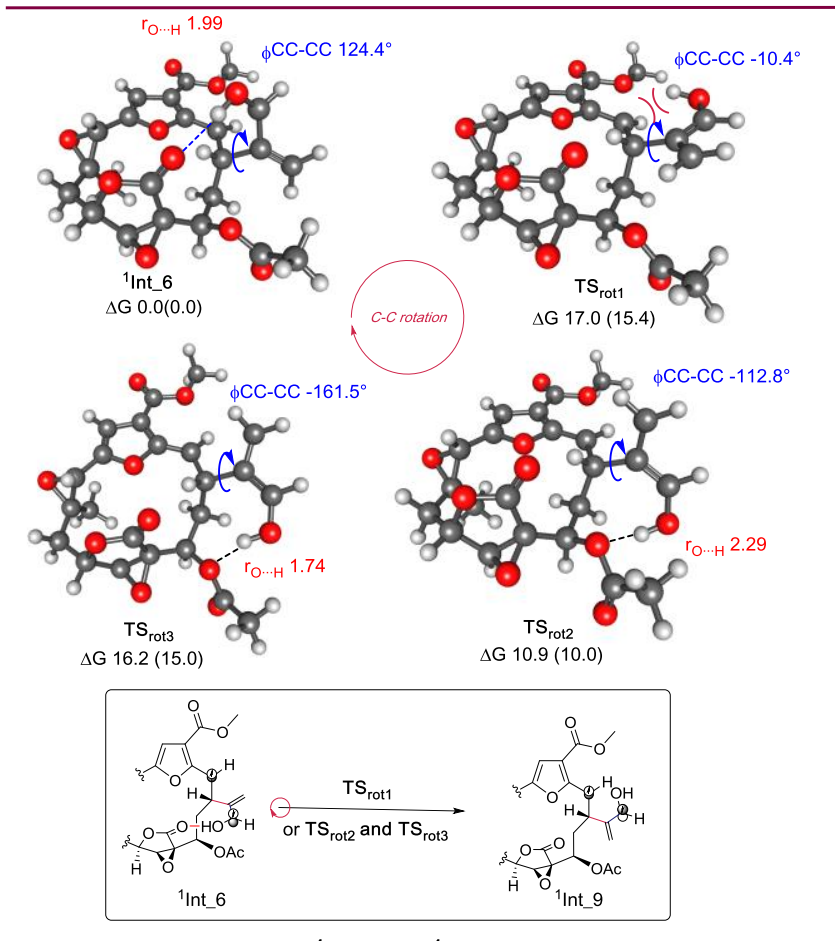

Figure 7. Interconversion of ${ }^{1}$ Int_6 6 and ${ }^{1}$ Int_9.

Summary

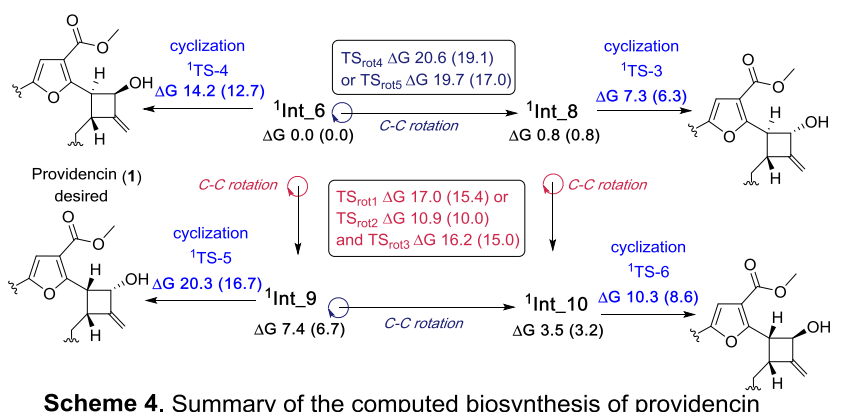

Following photoexcitation, irreversible hydrogen-abstraction in the triplet-state of bipinnatin $\mathrm{E}$ favors the formation of biradical intermediate ${ }^{1}$ Int_6. The presence of an intramolecular hydrogen bond and a congested steric environment stabilize this intermediate to such an extent that conformational interconversion via C-C rotation is prohibited and the Curtin-Hammett principle does not govern stereoselectivity. Radical recombination, forming the cyclobutane ring occurs readily, resulting in the correct stereochemistry of providencin (Scheme 4). Substrates without 
the ability to prevent conformational equilibration of the biradical intermediate are predicted to form cyclobutanes epimeric at C-17, in accordance with the Curtin-Hammett principle. At this level of calculation, it is reasonable to conclude that the biosynthetic proposal for the formation of providencin from bipinnatin E through a Norrish-Yang cyclisation reaction is feasible in nature and providencin is the expected product from the four possible diastereoisomers generated in the radical combination step. A synthetic work towards the synthesis of bipinnatin $E$ for the biomimetic synthesis of providencin using Norrish-Yang cyclisation reaction is also carrying out in our laboratory. Progress will be reported in due course.

\section{Acknowledgements}

This work is funded by National Science Foundation of China (NSFC) under the grant No 21502101 and NSF of Ningbo (2017A610070). We acknowledge the use of XSEDE through allocation TG-CHE180056 and the RMACC Summit supercomputer, supported by the National Science Foundation, the University of Colorado Boulder and Colorado State University. We are also grateful for access to the University of Nottingham High Performance Computing Facility. Thanks to the International Doctoral Innovation Centre, Ningbo Education Bureau, Ningbo Science and Technology Bureau, and the University of Nottingham Ningbo China.

\section{References}

(1) Marrero, J.; Rodríguez, A. D.; Baran, P.; Raptis, R. G. Org. Lett. 2003, 5 (14), 2551-2554.

(2) (a) Toelle, N.; Weinstabl, H.; Gaich, T.; Mulzer, J. Angew. Chem., Int. Ed. 2014, 53 (15), 3859-3862; (b) Gaich, T.; Weinstabl, H.; Mulzer, J.; Synlett, 2009, 9, 1357-1366.

(3) White, J. D.; Jana, S. J. Org. Chem. 2014, 79 (2), 700-710.

(4) White, J. D.; Jana, S. Org. Lett. 2009, 11 (6), 1433-1436.

(5) Stevens, S. J.; Bérubé, A.; Wood, J. L. Tetrahedron 2011, 67 (35), 6479-6481.

(6) Bray, C. D.; Pattenden, G. Tetrahedron Lett. 2006, 47 (23), 3937-3939.

(7) Rodriguez, A. D.; Shi, J.-G. J. Org. Chem. 1998, 63, 420421.

(8) Norrish, R. G. W.; Bamford, C. H. Nat. Chem. 1937, 140, 195-196.

(9) Yang, N. C.; Yang, D.-D. H. J. Am. Chem. Soc. 1958, 80 (3), 2913-2914.

(10) Yoshioka, S.; Nagatomo, M.; Inoue, M. Org. Lett. 2015, 17, 90-93.

(11) Hoffmann, N.; Hoffmann, N. Chem. Rev. 2008, 108 (3), 1052-1103.

(12) Zhou, J.; Zhang, J.; Cheng, A.; Xiong, Y.; Liu, L.; Lou, H. Org. Lett. 2015, 8-11.

(13) Paquette, L.A.; Sugimura T. J. Am. Chem. Soc. 1986, 108, 3841-3842.

(14) Sugimura, T.; Paquette, L. A. J. Am. Chem. Soc. 1987, 5, 3017-3024.

(15) Ignatenko, V. A.; Tochtrop, G. P. J. Org. Chem. 2013, 78 (8), 3821-3831.

(16) Bach, T.; Hehn, J. P. Angew. Chem., Int. Ed. 2011, 50 (5), $1000-1045$.
(17) Hong, Y. J.; Tantillo, D. J. Chem. Soc. Rev. 2014, 43 (14), 5042-5050.

(18) Peng, Q.; Paton, R. S. Acc. Chem. Res. 2016, 49 (5), 1042 1051.

(19) Cheng, G. J.; Zhang, X.; Chung, L. W.; Xu, L.; Wu, Y. D. J. Am. Chem. Soc. 2015, 137 (5), 1706-1725.

(20) Hong, Y. J.; Tantillo, D. J. Nat. Chem. 2009, 1 (5), 384-389.

(21) Hong, Y. J.; Tantillo, D. J. Nat Chem 2014, 6 (2), 104-111.

(22) Hornsby, C. E.; Paton, R. S. Nat. Chem. 2014, 6 (2), 88-89.

(23) Roethle, P. A.; Hernandez, P. T.; Trauner, D. Org. Lett. 2006, 8 (25), 5901-5904.

(24) Tang, B.; Bray, C. D.; Pattenden, G. Tetrahedron Lett. 2006, 47 (36), 6401-6404.

(25) Wang, S. C.; Tantillo, D. J. J. Org. chem., 2008, 73(4), 15161523.

(26) Stichnoth, D.; Kölle, P.; Kimbrough, T. J.; Riedle, E.; de Vivie-Riedle, R.; Trauner, D. Nat. Commun. 2014, 5, 5597.

(27) Li, Y.; Pattenden, G. Tetrahedron Lett. 2011, 52(26), 33153319.

(28) Li, Y.; Pattenden, G. Nat. Prod. Rep. 2011, 28(2), 429-440.

(29) Lygo, B.; Palframan, M. J.; Pattenden, G. Org. Biomol. Chem. 2014, 12, 7270-7278.

(30) Tang, B.; Simion, R.; Paton, R. S. Synlett 2015, 26(4), 501507.

(31) Calculations were performed with Gaussian09 rev. E01: see the SI for full details of the computatiaonl methods.

(32) Sinicropi, A.; Barbosa, F.; Basosi, R.; Giese, B.; Olivucci, M. Angew. Chem., Int. Ed. 2005, 44(16), 2390-2393. Tadi, J. M.; Xu, L.; Houk, K. N.; Moortgat, G. K. J. Org. Chem. 2011, 1614-1620.

Moskvin, A. F.; Yablonskii, O. P.; Bondar, L. F. Theor. Exp. Chem. 1968, 2(5), 469-472. 